\title{
Late time transition of Universe and the hybrid scale factor
}

\author{
E. Aydiner ${ }^{1, \mathrm{a}}$, I. Basaran-Öz ${ }^{1}$, T. Dereli ${ }^{2}$, M. Sarisaman ${ }^{1}$ \\ ${ }^{1}$ Department of Physics, Faculty of Science, İstanbul University, 34134 Istanbul, Turkey \\ ${ }^{2}$ Department of Physics, Koç University, Sarıyer, 34450 Istanbul, Turkey
}

Received: 7 December 2021 / Accepted: 4 January 2022 / Published online: 15 January 2022

(C) The Author(s) 2022

\begin{abstract}
In this study, we propose an interacting model to explain the physical mechanism of the late time transition from matter-dominated era to the dark energy-dominated era of the Universe evolution and to obtain a scale factor $a(t)$ representing two eras together. In the present model, we consider a minimal coupling of two scalar fields which correspond to the dark matter and dark energy interacting through a potential based on the FLRW framework. Analytical solution of this model leads to a new scale factor $a(t)$ in the hybrid form $a(t)=a_{0}\left(t / t_{0}\right)^{\alpha} e^{h t / t_{0}}$. This peculiar result reveals that the scale factor behaving as $a(t) \propto\left(t / t_{0}\right)^{\alpha}$ in the range $t / t_{0} \leq t_{c}$ corresponds to the matter-dominated era while $a(t) \propto \exp \left(h t / t_{0}\right)$ in the range $t / t_{0}>t_{c}$ accounts for the dark energy-dominated era, respectively. Surprisingly, we explore that the transition from the power-law to the exponential expansion appears at the crossover time $t_{0} \approx 9.8$ Gyear. We attain that the presented model leads to precisely correct results so that the crossover time $t_{0}$ and $\alpha$ are completely consistent with the exact solution of the FLRW and re-scaled Hubble parameter $H_{0}$ lies within the observed limits given by Planck, CMB and SNIa data (or other combinations), which lead to consistent cosmological quantities such as the dimensionless Hubble parameter $h$, deceleration parameter $q$, jerk parameter $j$ and EoS parameter $w$. We also discuss time dependent behavior of the dark energy and dark matter to show their roles on the time evolution of the universe. Additionally, we observe that all main results completely depend on the structure of the interaction potential when the parameter values are tuned to satisfy the zero energy condition. Finally, we conclude that interactions in the dark sector may play an important role on the time evolution and provides a mechanism to explain the late time transition of the Universe.
\end{abstract}

\footnotetext{
a e-mail: ekrem.aydiner@istanbul.edu.tr (corresponding author)
}

\section{Introduction}

Observations of Type Ia Supernova (SNIa) show that the expansion of Universe is accelerating faster than expected $[1,2]$. These observational evidences clearly indicate that Universe evolves from the matter-dominated to an accelerated expansion era. After this pioneering discovery, it has been suggested that dark energy (DE) which behaves like the opposite of gravity and has repulsive pressure is the source of this accelerated expansion of the universe and this phenomenon is called the late time transition of the universe evolution. At this point, two problems appear: The first problem is what is the cause or the physical mechanism of this transition? The second important problem is, can we express both the matter-dominant and dark-energy-dominant periods with a single scale factor? These are very important problems of the cosmology. In this study, we will focus on these two important problems of the cosmology.

Late time transition: The late time transition problem is one of the important problems of cosmology that has not been fully resolved yet. After observing that the universe is expanding at an accelerated rate, the existence of dark energy has been held responsible as the source of this expansion and many interesting DE models have been proposed in the literature such as quintessence [3], phantom [4], k-essence [5], tachyon [6], Chaplygin gas [7], holographic dark energy [8]. Although these models are the leading candidates for explaining the physical origin of the dark energy, they are far from explaining the physical mechanism of the late time transition. In fact, is this transition caused by the existence of dark energy? Even if dark energy can be considered to be the cause of late time exponential expansion of the Universe, the existence of dark energy alone does not seem to be sufficient to explain this transition. The other point is rather distinct from the first one where the key question is: Can we describe this critical transition as a phase transition or a catastrophic transition? If we view the problem in terms of the statistical mechanics of phase transitions, we can not say that it is a first 
or second-order phase transition. The turnover in the evolution process favors a catastrophic jump rather than a phase transition (Please see [9] for the catastrophic transitions). It is clear that the problem is more sophisticated, and requires an elaborate approach. Therefore, we may need new players to enlarge this discussion.

Hybrid scale factor: It is well-known that the evolution of Universe can be represented by the scale factor $a(t)$. Indeed, after early time inflation $[10,11]$ the scale factor takes the functional form of $a(t) \propto t^{1 / 2}$ and $a(t) \propto t^{2 / 3}$ for the radiation-dominated and the matter-dominated era, respectively. However, the dark energy era is represented by $a(t) \propto \exp \left(H_{0} t\right)$ where $H_{0}$ is the Hubble parameter. In theoretical studies, scale factors that explain these periods one by one have been suggested. However, a model representing all or at least two of these phases with a single scale factor has not yet been proposed. Furthermore, to the best of our knowledge, so far, a model that represents the matter-dominant period and the dark-energy-dominant period together with a single or hybrid scale factor has not yet been obtained, although numerous models have been proposed to explain this phenomenon, for instance, in Refs. [12-22]. Therefore, this theoretical problem deserves attention.

To summarize, so far, no model study has been conducted to explain the transition from the matter-dominant period to the dark-energy-dominant period with transition time in the cosmic time-line and to show that this transition will be represented by a single scale factor. Therefore, without understanding this critical transition problems, it seems to be unlikely to proceed toward a comprehensive theory of cosmology. Based on these motivations, in this study, we will focus on the late time transition of Universe and propose a new model to solve these challenging problems of the cosmology.

To solve these problems, one can consider that the possible candidates are baryonic or non-baryonic dark matter (DM) and dark energy. Indeed, it was recently suggested that dark energy could be dynamic, evolving with time [23-26]. Clearly, one can state that a single fluid with a constant cannot give rise to a realistic cosmic history. Therefore, the realistic Universe model should be dominated by more ingredients, which can be defined by different EoS parameters [27]. Indeed, it is shown in the literature that the interacting models have potential to solve many problems of the cosmology. For example, many interacting models have been used to solve the singularity and cosmic coincidence problems [2851]. More recently, a different interaction model has been introduced by Aydiner in Ref. [52]. In his study, it has been shown that the interaction between matter, dark matter and dark energy has led to the chaotic evolution of the Universe. It was seen that this model combined the big-bang model and the oscillatory Universe models, as well as had the potential to solve many fundamental problems of cosmology such as singularity, the future of the Universe, the formation of the galaxies and large-scale organization of the Universe. However, the late time transition was not specifically discussed by Aydiner in Ref. [52] and in the others [28-51].

These studies provide a possible solution to explain the mechanism of the late time transition of the Universe based on interactions between dark energy and dark matter. Therefore, our aim in this study is to discuss the late time transition of the Universe based on the interaction of dark matter and dark energy. Here, for simplicity, we define these components as the two different scalar fields in the theoretical framework of the FLRW metric. We consider the interaction between them and generalize the model based on the motivation in Ref. [53]. In their study, Dereli and Tucker describe classical models of gravitation interacting with scalar fields whose solutions involve degenerate metrics. They show that some of these solutions exhibit transitions from a Euclidean domain to a Lorentzian space-time corresponding to a spatially flat FLRW cosmology. Inspired from this study, here, we generalized this method to two interacting scalar field model which corresponds to the dark matter and dark energy interaction in a flat FLRW cosmology. We assume that the two scalar fields interact with a potential based on two oscillator and two anti-oscillator to satisfy the Hamiltonian zero energy condition and Einstein field equations.

Here solving Einstein field equations we analytically obtain a hybrid scale factor as $a(t)=a_{0}\left(t / t_{0}\right)^{\alpha} e^{h t / t_{0}}$ which represents both the matter dominated era and dark energy era. We show that time crossover appear around $t c \approx 9.8$ Gyear, which indicates transition time from the matter dominated era to the dark energy era. We find the parameter $\alpha$ is equal to $2 / 3$ and the Hubble parameter which is around $H_{0}=69.5$ and $H_{0}=73.5 \mathrm{~km} \mathrm{~s}^{-1} \mathrm{Mpc}^{-1}$ depends on parameters of the interacting potential. Additionally, we compute the other cosmological parameters such as dimensionless Hubble $h$, dimensionless deceleration $q$, dimensionless equation of states (EoS) $w$ and dimensionless jerk parameters $j$ for this model. We show that our theoretical results are consistent with previous theoretical results $[54,55]$ and all cosmological observations such as CMB with Planck [56-58], CMB without Planck [59-61], No CMB, with BBN [6264], Cepheids-SNIa [65-74], and other combinations given in Ref. [75] (and references therein).

The outline of the paper is organized as follows. In Sect. 2, we present the two-scalar cosmology model with Lagrangian in the FLRW framework. In Sect. 3, we define an interaction potential and analytically obtain an hybrid scale factor $a(t)$ from the Lagrangian solutions. In Sect. 4, we numerically analyze the scale factor $a(t)$ and we show that presence of the transition matter dominated era to the dark energy dominated era. In Sect. 5, we numerically obtain the other cosmological quantities and discuss their time-dependent characteristic 
behaviors. In Sect. 6, we discuss the limit behaviours of all quantities. In Sect. 7, we discuss the time dependent behavior of the dark energy and dark matter. Finally, we present the conclusion and important remarks of this study in the last section.

\section{Interaction between DM and DE}

In this study, we propose that DM and DE can be represented by two different scalar fields for instance $\phi$ and $\sigma$, and they interacts with a potential. In this case, the action of minimally coupled scalar gravity for two-scalar fields is described by

$$
\begin{aligned}
S= & \int d^{4} x \sqrt{-g} \\
& \times\left[\frac{R}{2 \kappa}+\frac{1}{2}\left(\partial_{\mu} \phi \partial^{\mu} \phi+\partial_{\mu} \sigma \partial^{\mu} \sigma\right)-V(\phi, \sigma)\right],
\end{aligned}
$$

where $\phi, \sigma: \mathbb{R}^{4} \rightarrow \mathbb{R}$ are scalar-valued $C^{\infty}$ fields, $V$ is the potential expressed as a function of the scalar fields, $R$ is the Ricci scalar, and $\kappa=8 \pi G / c^{4}$ is a constant that we use the geometric unit system, i.e. $\kappa=1$. Scalar fields are defined on a manifold with metric $\gamma_{a b}(\phi, \sigma)$ and action is invariant under the symmetries of the scalar fields.

Consider the FLRW metric expressing a homogeneous isotropic space-time metric given by

$d s^{2}=h_{\mu \nu} d x^{\mu} d x^{\nu}$

with the metric $h_{\mu v}=\operatorname{diag}\left(-1, a(t) I_{3}\right), I_{3}=\operatorname{diag}(1,1,1)$ is $3 \times 3$ identity matrix, $a(t): \mathbb{R} \rightarrow \mathbb{R}$ is a differentiable function which is known as time-dependent scale factor. The Ricci scalar equipped with this space-time (2) is specified by

$R=6\left(\frac{\ddot{a}}{a}+\frac{\dot{a}^{2}}{a^{2}}\right)$

where the dot denotes the derivative with respect to time. Now, the point-like Lagrangian for DM and DE interaction can be written as follow

$\mathcal{L}=-3 a \dot{a}^{2}+\frac{a^{3}}{2}\left(\dot{\phi}^{2}+\dot{\sigma}^{2}\right)-a^{3} V(\phi, \sigma)$.

At this point, we can obtain analytical solution using the Lagrangian (4).

\section{Analytical results}

One can easily reveal the set of equations of motion by means of the dynamical variables $\{a, \phi, \sigma\}$ for the Lagrangian (4). These are obtained as

$2 \frac{\ddot{a}}{a}+\frac{\dot{a}^{2}}{a^{2}}+\frac{1}{2}\left(\dot{\phi}^{2}+\dot{\sigma}^{2}\right)-V(\phi, \sigma)=0$,

$$
\begin{aligned}
& \ddot{\phi}+3 \frac{\dot{a}}{a} \dot{\phi}+\frac{\partial V(\phi, \sigma)}{\partial \phi}=0, \\
& \ddot{\sigma}+3 \frac{\dot{a}}{a} \dot{\sigma}+\frac{\partial V(\phi, \sigma)}{\partial \sigma}=0 .
\end{aligned}
$$

Notice that once we impose the zero energy condition, the remaining equation necessary for this theory is obtained as follows

$$
-3 \frac{\dot{a}^{2}}{a^{2}}+\frac{1}{2}\left(\dot{\phi}^{2}+\dot{\sigma}^{2}\right)+V(\phi, \sigma)=0 .
$$

To solve the equations of motion in Eqs. (5) and (6) we need to determine an appropriate potential which correspond to the two oscillators and two anti-oscillators. In our study, we focus our attention on a specific potential characterized by

$$
\begin{aligned}
& 2 \alpha^{2}\left(X_{1}^{2}-X_{2}^{2}+Y_{1}^{2}-Y_{2}^{2}\right) V(\phi, \sigma) \\
& =A_{1} X_{1}^{2}+A_{2} X_{2}^{2}+B_{1} Y_{1}^{2}+B_{2} Y_{2}^{2} \\
& \quad+2 k_{1} X_{1} Y_{2}-2 k_{2} X_{2} Y_{1}
\end{aligned}
$$

where $A_{j}, B_{j}$ and $k_{j}(j=1,2)$ are interaction parameters.

Based on our experiences we know that this potential linearizes the field equations to get the precise solutions. However, in order to see that this potential gives rise to the physically meaningful and stable solutions, we have to check the potential surface. Therefore, inspired by the mechanical analogy, we introduce the following transformations

$$
\begin{aligned}
& X_{1}=a^{3 / 2} \cosh (\alpha \phi), \\
& X_{2}=a^{3 / 2} \sinh (\alpha \phi), \\
& Y_{1}=a^{3 / 2} \cosh (\alpha \sigma), \\
& Y_{2}=a^{3 / 2} \sinh (\alpha \sigma),
\end{aligned}
$$

where $\phi, \sigma \in[-\infty, \infty]$ and $a: \mathbb{R} \rightarrow \mathbb{R}^{+}$. By using these transformations, we can write the potential $V(\phi, \sigma)$ which explicitly depends on the scalar fields $\phi$ and $\sigma$ as

$$
\begin{aligned}
V(\phi, \sigma)= & \frac{1}{2 \alpha^{2}}\left[A_{1} \cosh ^{2}(\alpha \phi)+A_{2} \sinh ^{2}(\alpha \phi)\right. \\
& +B_{1} \cosh ^{2}(\alpha \sigma)+B_{2} \sinh ^{2}(\alpha \sigma) \\
& +k_{1} \cosh (\alpha \phi) \sinh (\alpha \sigma) \\
& \left.-k_{2} \cosh (\alpha \sigma) \sinh (\alpha \phi)\right]
\end{aligned}
$$

where the parameters are given for $\theta=\pi / 4$ and $\psi=\pi / 4$ as

$$
\begin{aligned}
& A_{1}=\Lambda_{1} \cos ^{2} \theta+\Lambda_{4} \sin ^{2} \theta, \\
& A_{2}=\Lambda_{2} \cos ^{2} \psi+\Lambda_{3} \sin ^{2} \psi, \\
& B_{1}=\Lambda_{2} \sin ^{2} \theta+\Lambda_{3} \cos ^{2} \theta, \\
& B_{2}=\Lambda_{1} \sin ^{2} \theta+\Lambda_{4} \cos ^{2} \theta, \\
& k_{1}=\left(\Lambda_{1}-\Lambda_{4}\right) \sin \theta \cos ^{2} \theta, \\
& k_{2}=\left(\Lambda_{2}-\Lambda_{3}\right) \sin \psi \cos ^{2} \psi,
\end{aligned}
$$

where $\Lambda_{i}(i=1, . ., 4)$ are also interaction constants. Employing the coordinate transformation to Eq. (6) we can 


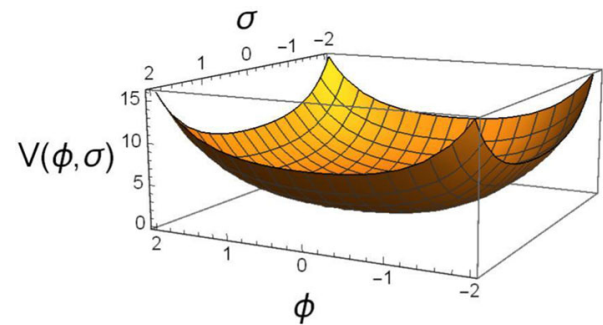

Fig. 1 The potential surface of $V$ as a function of field variables $\phi$ and $\sigma$. We set values as $A_{1}=B_{2}=1.005, A_{2}=B_{1}=2.005, k=0.005$

write the Hamiltonian constraint in the form

$$
\begin{aligned}
& \dot{X}_{1}^{2}-\dot{X}_{2}^{2}+\dot{Y}_{1}^{2}-\dot{Y}_{2}^{2}-A_{1} X_{1}^{2}-A_{2} X_{2}^{2}-B_{1} Y_{1}^{2}-B_{2} Y_{2}^{2} \\
& \quad-2 k_{1} X_{1} Y_{2}+2 k_{2} X_{2} Y_{1}=0 .
\end{aligned}
$$

From this equation, we find relation between parameters as $A_{1} \neq \pm B_{2}, A_{3} \neq \pm B_{1}, A_{1}+A_{2}=B_{1}+B_{2}$, $k_{1}=-1 / 2\left(A_{1}+B_{2}\right)$ and $k_{2}=-1 / 2\left(A_{2}+B_{1}\right)$. These relations satisfy Hamiltonian constraint equations.

On the other hand, here, for the sake of simplicity and analyse of the minima of the potential, we set $k_{1}=-k_{2}=k$. Then, the potential becomes

$$
\begin{aligned}
& V(\phi, \sigma)=\frac{1}{2 \alpha^{2}}\left[\delta_{1}+\delta_{2} \sinh ^{2}(\alpha \phi)+\delta_{3} \sinh ^{2}(\alpha \sigma)\right. \\
& \quad+2 k \sinh [\alpha(\sigma-\phi)]]
\end{aligned}
$$

where $\delta_{1}=\left(A_{1}+B_{1}\right), \delta_{2}=\left(A_{1}+A_{2}\right), \delta_{3}=\left(B_{1}+B_{2}\right)$ and $\alpha^{2}=3 / 4$. Furthermore, the potential $V(\phi, \sigma)$ should have natural identifications for small $\phi$ and $\sigma$. Therefore, we realize that the coefficients of $\phi^{2} / 2$ and $\sigma^{2} / 2$ terms can be identified by the positive-valued mass terms $m_{\phi}^{2}$ and $m_{\sigma}^{2}$ respectively, and $V(0,0)$ by the cosmological constant $\Lambda$. Potential can be expanded Taylor series up to the order of fifth terms as follows

$$
\begin{aligned}
V(\phi, \sigma)= & \frac{1}{2 \alpha^{2}}\left(A_{1}+B_{1}\right)+\frac{k}{\alpha}(\sigma-\phi)+\frac{1}{2}\left(A_{1}+A_{2}\right) \phi^{2} \\
& +\frac{1}{2}\left(B_{1}+B_{2}\right) \sigma^{2}+\frac{k \alpha}{2}\left(\phi^{2} \sigma-\sigma^{2} \phi\right) \\
& +\frac{k \alpha}{6}\left(\sigma^{3}-\phi^{3}\right)+\frac{k \alpha^{3}}{12}\left(\sigma^{3} \phi^{2}-\sigma^{2} \phi^{3}\right) \\
& +\mathcal{O}_{6}(\phi, \sigma),
\end{aligned}
$$

where $\mathcal{O}_{6}$ denotes the sixth and higher-order terms. We realize that this potential involves the associated potential terms in the catastrophic theory for small field variables.

In view of these results, the potential surface corresponding to $V$ governed by the field variables $\phi$ and $\sigma$ is displayed in Fig. 1. It is obvious that the potential surface has a global minimum in the limit of $k \rightarrow 0$ and $\nabla V(0,0) \rightarrow 0$. This minima guarantees that the solutions in Eq. (14) are stable.

The stable solutions arise around the minimal potential. These stable solutions also give rise to the stable cosmolog- ical solutions. Therefore, we see that in the limit of $k \rightarrow 0$, we get $\nabla V(0,0) \rightarrow 0$ for the appropriate parameter values. Based on this idea, the cosmological constant and the mass parameters of the scalar fields are obtained respectively as,

$$
\begin{aligned}
\Lambda & :=V(0,0)=\frac{2}{3}\left(A_{1}+B_{1}\right), \\
m_{\phi}^{2} & :=\partial_{\phi}^{2} V(0,0)=\left(A_{1}+A_{2}\right), \\
m_{\sigma}^{2} & :=\partial_{\sigma}^{2} V(0,0)=\left(B_{1}+B_{2}\right) .
\end{aligned}
$$

Using the expression in (7) and the transformations in (8), new Lagrangian can be written as

$$
\begin{aligned}
\mathcal{L}= & \dot{X}_{1}^{2}-\dot{X}_{2}^{2}+\dot{Y}_{1}^{2}-\dot{Y}_{2}^{2}+A_{1} X_{1}^{2}+A_{2} X_{2}^{2} \\
& +B_{1} Y_{1}^{2}+B_{2} Y_{2}^{2}+2 k_{1} X_{1} Y_{2}-2 k_{2} X_{2} Y_{1} .
\end{aligned}
$$

Thus, by linearization, instead of non-linear field equations in (5), four linear field equations are obtained as follows

$\ddot{X}_{1}=A_{1} X_{1}+k_{1} Y_{2}$,

$\ddot{X}_{2}=-A_{2} X_{2}+k_{2} Y_{1}$,

$\ddot{Y}_{1}=B_{1} Y_{1}-k_{2} X_{2}$,

$\ddot{Y}_{2}=-B_{2} Y_{2}-k_{1} X_{1}$.

These equations can be expressed in a more compact form with the identification

$\xi=\left(\begin{array}{c}X_{1} \\ X_{2} \\ Y_{1} \\ Y_{2}\end{array}\right)$

such that (16) amounts to the following equation

$\ddot{\xi}=\mathbb{M} \xi$,

where $\mathbb{M}$ is the matrix obtained from the coefficients of field equations in (16). To find an appropriate solution of the Eq.(18), we first find out the eigenvalues and eigenvectors which are obtained by the characteristic equation $\operatorname{det}(\mathbb{M}-\lambda \mathbb{I})=0$. Thus, corresponding eigenvalues are attained as follows

$$
\begin{aligned}
& \lambda_{1,2}=\frac{1}{2}\left[A_{1}-B_{2} \pm \sqrt{\left(A_{1}+B_{2}\right)^{2}-4 k_{1}^{2}}\right], \\
& \lambda_{3,4}=\frac{1}{2}\left[-A_{2}+B_{1} \pm \sqrt{\left(A_{2}+B_{1}\right)^{2}-4 k_{2}^{2}}\right],
\end{aligned}
$$

where we denote the distinct eigenvalues by means of " \pm " sign elements. The corresponding eigenvectors can be obtained accordingly

$$
\begin{aligned}
& S_{1}\left(\lambda_{i}\right)=B_{2}+\sum_{i=1}^{4} \lambda_{i}-k_{1}, \\
& S_{2}\left(\lambda_{i}\right)=B_{1}-\sum_{i=1}^{4} \lambda_{i}+k_{2},
\end{aligned}
$$


$S_{3}\left(\lambda_{i}\right)=A_{2}+\sum_{i=1}^{4} \lambda_{i}+k_{2}$,

$S_{4}\left(\lambda_{i}\right)=A_{1}-\sum_{i=1}^{4} \lambda_{i}-k_{1}$.

As a result, eigenvalues in Eq.(19) and eigenvectors in Eq. (20) give rise to the exact solutions of the field equations, which take the following form in components

$\xi_{i}=\sum_{i, j} S_{i}\left(\lambda_{j}\right)\left(\alpha_{j}+\beta_{j}\right)$

where $\alpha_{j}=m_{j} e^{\sqrt{\lambda_{j}} t}, \beta_{j}=n_{j} e^{-\sqrt{\lambda_{j}} t}, m_{j}$ and $n_{j}$ are constants and $i, j=1,2,3,4$. It is to be not that here $t$ is assumed as dimensionless parameter.

In view of these findings, we now return to our main discussion to find the solutions of cosmological quantities. At this point, we can give solutions to cosmological quantities for the Lagrangian in Eq. (4) with two scalar fields. In fact, the basic cosmological parameter is the scale factor $a(t)$ that is completely independent of position or direction and tells us how the expansion or contraction of the Universe depends on the cosmic time. We can present the scalar factor $a(t)$ in terms of the new coordinate variables as

$a(t)=\left[\frac{1}{2}\left(\xi_{1}^{2}-\xi_{2}^{2}+\xi_{3}^{2}-\xi_{4}^{2}\right)\right]^{\frac{1}{3}}$.

On the other hand, the scalar fields $\phi$ and $\sigma$ that appear in the Lagrangian which represent dark matter and dark energy, respectively, are given in the form:

$\phi(t)=\frac{1}{\alpha} \tanh ^{-1}\left(\frac{\xi_{2}}{\xi_{1}}\right), \quad \sigma(t)=\frac{1}{\alpha} \tanh ^{-1}\left(\frac{\xi_{4}}{\xi_{3}}\right)$.

Other cosmological parameters such as dimensionless Hubble $h$, deceleration $q$ and jerk $j$ parameters can be expressed in terms of $a, \dot{a}, \ddot{a}$ and $\dddot{a}$ as follows

$H:=\frac{\dot{a}}{a}, \quad q:=-\frac{\ddot{a}}{a H^{2}}, \quad j:=\frac{\dddot{a}}{a H^{3}}$.

These parameters can be determined from Taylor's expansion of the scale factor $a(t)$. Here, by definition, the Hubble parameter $H$ tells us the cosmic time-dependent expansion rate of the Universe, the Deceleration parameter $q$ tells us the change in the expansion rate of the Universe and the Jerk parameter $j$ tells us the change in the acceleration or deceleration of the Universe. Additionally, the effective equation of state (EoS) parameter is given in terms of the effective pressure $p_{\text {eff }}$ and effective density $\rho_{\text {eff }}$ as follows

$w_{e f f}=\frac{p_{e f f}}{\rho_{e f f}}$, where the pressure is given by the expression $p_{\text {eff }}=\frac{1}{2} \dot{\phi}^{2}+$ $\frac{1}{2} \dot{\sigma}^{2}-V(\phi, \sigma)$, whereas the density is provided by $\rho_{\text {eff }}=$ $\frac{1}{2} \dot{\phi}^{2}+\frac{1}{2} \dot{\sigma}^{2}+V(\phi, \sigma)$.

\section{Numerical results of the scale factor}

We solve field equations in Eq. (16) and analytically obtain the scale factor $a(t)$ in Eq. (22). Now, we give the numerical result of the scale factor in Fig. 2 for relatively weak and relatively strong interactions. In this numerical solutions, we set parameters arbitrarily as $\Lambda_{1}=0.05, \Lambda_{2}=2.5, \Lambda_{3}=$ $0.5, \Lambda_{4}=2.05, A_{1}=A_{4}=1.05, A_{2}=A_{3}=1.5, k_{1}=$ $-1.0, k_{2}=1.0$ for the red circle line; $\Lambda_{1}=0.1, \Lambda_{2}=2.6$, $\Lambda_{3}=0.6, \Lambda_{4}=2.2, A_{1}=A_{4}=1.15, A_{2}=A_{3}=1.6$, $k_{1}=-1.05, k_{2}=1.0$ for the green star line, where here and in what follows, these parameters are selected such that minimal and stable potential together with minimal/maximal interactions for small/large $k$ values are guaranteed.

The numerical solution of the dimensionless scale factor in Eq. (22) versus scaled axes $t / t_{0}$ is given in Fig. 2a. However, the dimensionless scale factor is provided by $\log -\log$ and semi-log scale in Fig. 2b and (c), respectively. When we fit the data of the dimensionless scale factor in Fig. 2a we see that our data give a hybrid relation as

$a(t)=\left[\frac{1}{2}\left(\xi_{1}^{2}-\xi_{2}^{2}+\xi_{1}^{2}-\xi_{2}^{2}\right)\right]^{\frac{1}{3}}=a_{0}\left(\frac{t}{t_{0}}\right)^{\alpha} e^{h \frac{t}{t_{0}}}$.

where little $h$ is the dimensionless Hubble parameter [55, $76,77]$ and $t_{0}$ is a constant. It first time, we precisely obtain a hybrid scale factor by using an interacting model. This is very interesting and amazing result. We show that an scale factor cover, at the same time, the power-law and exponential behavior without using any approximation or ansatz. Furthermore, this result provide that our model can explain the time evolution of the matter dominate era and dark energy dominated era. On the other hand, to obtain detail results and to determine parameter of the scale factor in Eq. (26) we plot $\log -\log$ and semi-log of this quantity in n Fig. 2b, c. It can be observed from the log-log plot in Fig. $2 b$ that the scale factor increases by a power-law up to a crossover time $t_{c}$ point. On the other hand, above this critical point, it increases exponentially as seen in Fig. 2c. This crossover point indicates the transition from power-law expansion to the exponentially expanding era of the Universe. This extraordinarily important and surprising result solves the late-time transition problem which is one of the most important problems of cosmology. Our numerical results clearly show that the transition in the scale factor $a(t)$ can be represented by

$a(t) \propto\left\{\begin{array}{l}\left(t / t_{0}\right)^{\alpha} \text { for } t / t_{0} \leq t_{c}, \\ e^{h\left(t / t_{0}\right)} \text { for } t / t_{0}>t_{c},\end{array}\right.$ 

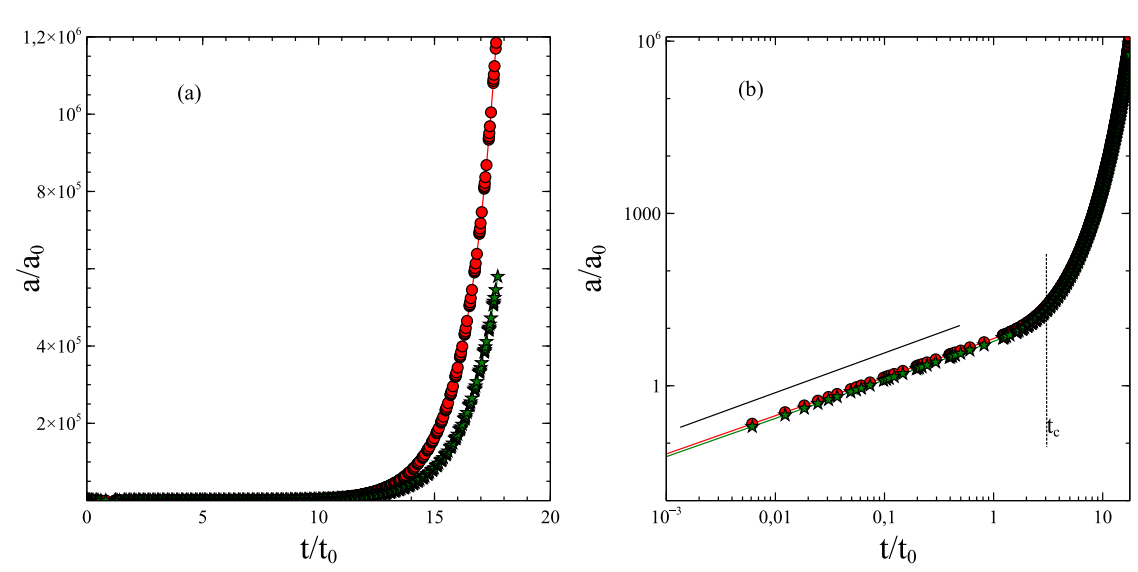

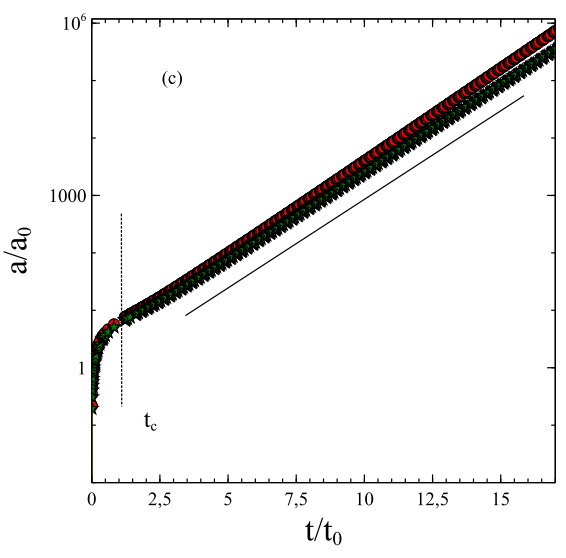

Fig. 2 In a, the scale factor $a(t)$ with respect to the cosmic time is given. Here we set the parameter values $\Lambda_{1}=0.05, \Lambda_{2}=2.5$, $\Lambda_{3}=0.5, \Lambda_{4}=2.05, A_{1}=A_{4}=1.05, A_{2}=A_{3}=1.5, k_{1}=-1.0$, $k_{2}=1.0$ for the red circle line; $\Lambda_{1}=0.1, \Lambda_{2}=2.6, \Lambda_{3}=0.6$,

where $t_{c}$ is also dimensionless parameter. We will discuss the $t_{c}$ below.

We numerically solved Eq. (22) for various interacting parameters, and, interestingly we found power-law exponent as $\alpha=2 / 3$ which is consistent with the Einstein-de Sitter solution of the Friedman equations for the matter dominated era. It is very consistent with the theoretical solutions [54]. This result denotes that matter dominated era evaluates with time $\left(t / t_{0}\right)^{2 / 3}$ for the $t \leq t_{c}$. On the other hand, we solved Eq. (22) for various interacting parameters, and we find that time the second term dominates the solution of scale factor $a(t)$ for the $t / t_{0}>t_{c}$ as seen from Fig. 2c. For example, for different two data set we plot the Fig. 2 and, in our analyses, surprisingly, we find that the dimensionless scale factor takes value between $h=0.695$ and $h=0.735$ around depend on interactions parameters. It is know that the time dependent Hubble parameter is defined as $H_{0}=100 \mathrm{~h} \mathrm{~km}$ $\mathrm{s}^{-1} \mathrm{Mpc}^{-1}[55,76,77]$. According this definition the timedependent Hubble parameters correspond to $H_{0}=69.5$ and $H_{0}=73.5 \mathrm{~km} \mathrm{~s}^{-1} \mathrm{Mpc}^{-1}$ for $h=0.695$ and $h=0.735$, respectively. These theoretical results are completely agree with the observational results CMB with Planck [56-58], CMB without Planck [59-61], No CMB, with BBN [6264], and Cepheids-SNIa [65-74]. It is assume that the current value in the late time inflation phase is about $H_{0}=70.88$ $\mathrm{km} \mathrm{s}^{-1} \mathrm{Mpc}^{-1}$ due to Planck and SNIa observations. In the numerical procedure, we used arbitrary parameter values and we see that choosing different parameter values does not change the character of the solution in Eq. (26). However, we see that choosing arbitrary parameters change, particularly, the slope of Fig. 2b, c. According to our findings, we show that we can explain the late time crossover from power-law to exponential expansion of the Universe by using the FLRW model including DM and DE interactions. Furthermore, we
$\Lambda_{4}=2.2, A_{1}=A_{4}=1.15, A_{2}=A_{3}=1.6, k_{1}=-1.05, k_{2}=1.0$ for the green star line. In $\mathbf{b}$, scale factor in $\log -\log$ scale is given. In (c), scale factor $a(t)$ in semi-log plot is displayed. Here $t_{c}$ value is given by Eq. (28)

explicitly obtain a real scale factor involving power and exponential terms in a single formula from the model. We report these results for the first time by using a model-dependent study.

In this section, finally, we discuss the $t_{c}$ and $t_{0}$. The crossover time $t_{c}$ can be approximately estimated from Fig. 2b, c as between 1.3 and 1.5. However, we know that the crossover time is equal to $t_{c}=t / t_{0}$ and which can be precisely obtained by using the relation $t_{c}^{2 / 3}=e^{h t_{c}}$. Thus, $t_{c}$ can be determined by the following equation

$\frac{\ln t_{c}}{t_{c}}=\frac{3 h}{2}$.

For the dimensionless Hubble parameter $h \simeq 0.7$ and $t \simeq 14$ Gyear this relation gives $t_{c} \simeq 1.428$ Gyear which provides that the value of the $t_{0}$ is $t_{0}=1 / H_{0}=9.8$ Gyear which refers to Einsetin-de Sitter solution [54] (See also Eq. (6.33) in Ref. [55]. This is another very important result of the model. Thus, the model we propose also predicts the transition from matter dominated era to the dark energy era perfectly with full precision.

\section{Numerical results of other kinematic parameters}

In this section, we numerically obtain the dimensionless kinetic parameters such as Hubble parameter $h$, the deceleration parameter $q$, The jerk parameter $j$ and the EoS parameter for relatively weak and relatively strong interactions. All of these parameters can be obtained from the scale factor $a$ we obtained. In these numerical solutions, we set parameters arbitrarily as $\Lambda_{1}=0.05, \Lambda_{2}=2.5, \Lambda_{3}=0.5, \Lambda_{4}=2.05$, $A_{1}=A_{4}=1.05, A_{2}=A_{3}=1.5, k_{1}=-1.0, k_{2}=1.0$ for the red circle line; $\Lambda_{1}=0.1, \Lambda_{2}=2.6, \Lambda_{3}=0.6$, 

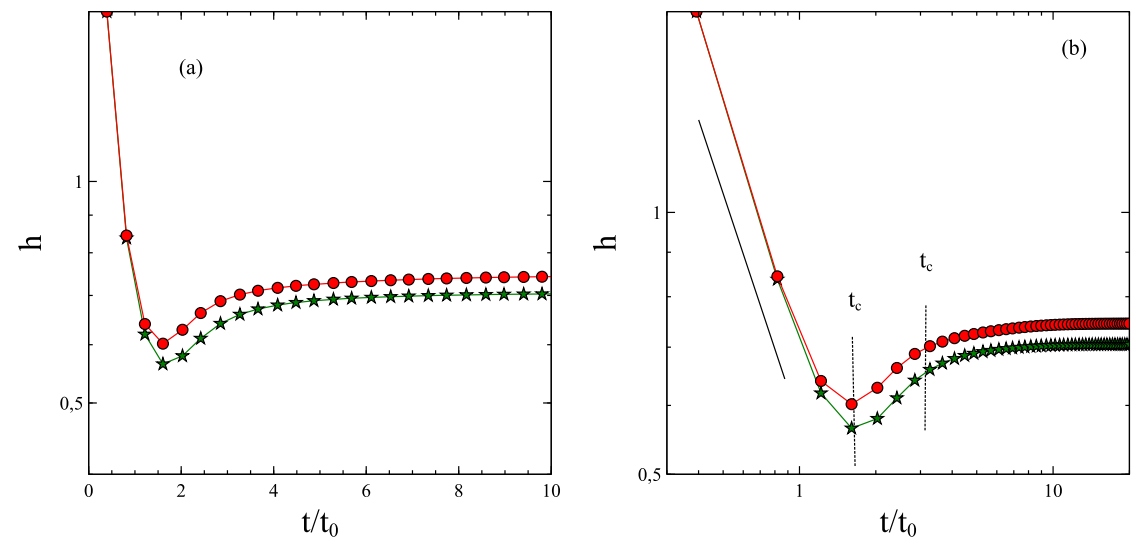

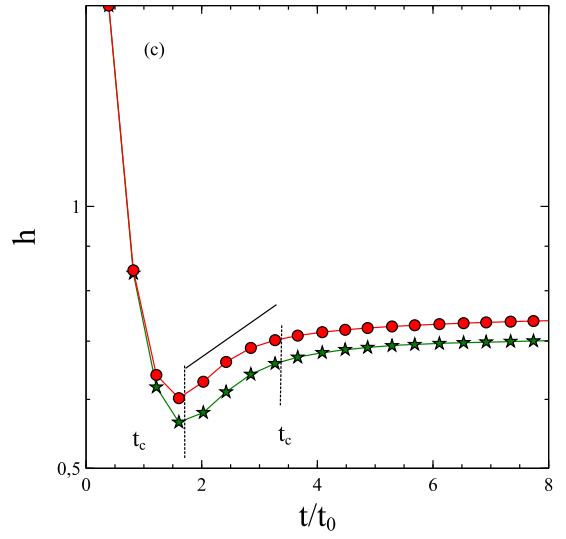

Fig. 3 In a, the dimensionless Hubble parameter $h$ with respect to cosmic time is given. Here we set the parameter values $\Lambda_{1}=0.05$, $\Lambda_{2}=2.5, \Lambda_{3}=0.5, \Lambda_{4}=2.05, A_{1}=A_{4}=1.05, A_{2}=A_{3}=1.5$, $k_{1}=-1.0, k_{2}=1.0$ for the red circle line; $\Lambda_{1}=0.1, \Lambda_{2}=2.6$,
$\Lambda_{3}=0.6, \Lambda_{4}=2.2, A_{1}=A_{4}=1.15, A_{2}=A_{3}=1.6, k_{1}=-1.05$, $k_{2}=1.0$ for the green star line. In $\mathbf{b}$, Hubble parameter $h$ in Log-Log scale is displayed. In c, dimensionless Hubble parameter $h$ in semi-log plot is shown
$\Lambda_{4}=2.2, A_{1}=A_{4}=1.15, A_{2}=A_{3}=1.6, k_{1}=-1.05$, $k_{2}=1.0$ for the green star line in all figures below. Here, our aim is to show the detail analysing of the characterising behaviour of these quantities and to provide that results of the model are consistent with the observational data.

\subsection{The dimensionless Hubble parameter $h$}

The Hubble parameter is given by the ratio of the rate of change of the scale factor to the current value of the scale factor $a$, which reflects the characteristic rate of the expansion of the Universe. The Hubble parameter can be obtained by using observational data, which depends on the red-shift. It takes different values for the radiation-dominated era, the matterdominated era and late time inflation. In our case, Hubble parameter is obtained from the model. The time dependence of the dimensionless Hubble parameter for the present model is provided in Fig. 3. The time-dependent behavior of the dimensionless Hubble parameter is displayed in Fig. 3a. However, the Hubble parameter is given by log-log and semilog scales in Fig. 3b, c, respectively.

It is to be noticed that the Hubble parameter has an anomaly depending on the scale factor $a$. It starts from a maximum value and rapidly drops to a minimum value, and then reaches up to a maximum value with time. This minima corresponds to the critical transition time $t_{c}$ observed in scale factor behavior. Clearly, we expect the dramatic change of the Hubble parameter in the case of the phase-like catastrophic transition from matter dominate era to dark energy dominate era. However, this minima additionally emphasizes that before the catastrophic transition, there occurs a short deceleration in the expansion of the Universe. This is a very interesting point from which its physical meaning and mechanism can be discussed profoundly. In order to see some more details of the evolution of the dimensionless Hubble parameter, one can analyze the Fig. 3b, c further. In Fig. 3b, it is seen that the Hubble parameter decreases as $h \propto\left(t / t_{0}\right)^{-\tilde{\alpha}}$ up to the critical time point $t_{c}$. On the other hand, above $t_{c}$, it increases exponentially as $h \propto e^{\tilde{\alpha}^{\prime}\left(t / t_{0}\right)}$ and it reaches up to a constant value, as observed, for relatively weak and relatively strong interactions, where $\tilde{\alpha}$, and $\tilde{\alpha}^{\prime}$ denote arbitrary constant parameters. The constant value of the dimensionless Hubble parameter $h$ for our interaction parameter set refers to $H_{0}=69.5$ and $H_{0}=73.5 \mathrm{~km} \mathrm{~s}^{-1} \mathrm{Mpc}^{-1}$ for $h=0.695$ and $h=0.735$, respectively as seen in Fig. 3. This numerical solution shows the characteristic behaviour of the dimensionless Hubble parameter $h$, at the same time it provides that our model produce quite consistent result with observational data for the different cosmological eras of the Universe. Furthermore, it denotes the presence of the an anomaly at around the transition from the matter dominated era to the dark energy dominated era.

\subsection{The dimensionless deceleration parameter $q$}

The deceleration parameter $q$ in cosmology is a dimensionless measure of the cosmic acceleration of the expansion of space in a FLRW Universe. In general, $q$ takes a negative sign and varies with the cosmic time, except in a few special cosmological models. Except in the speculative case of phantom energy, all postulated forms of mass-energy yield a deceleration parameter $q \geq-1$. On the other hand, for any non-phantom Universe, there must be a decreasing Hubble parameter, except in the case of the distant future of a $\Lambda \mathrm{CDM}$ model, where $q$ goes to -1 from above and the Hubble parameter gets asymptote to a constant value of $H_{0} \simeq \sqrt{\Lambda / 3}$. In our case, the deceleration parameter is obtained from the model itself. The time dependence of the deceleration param- 

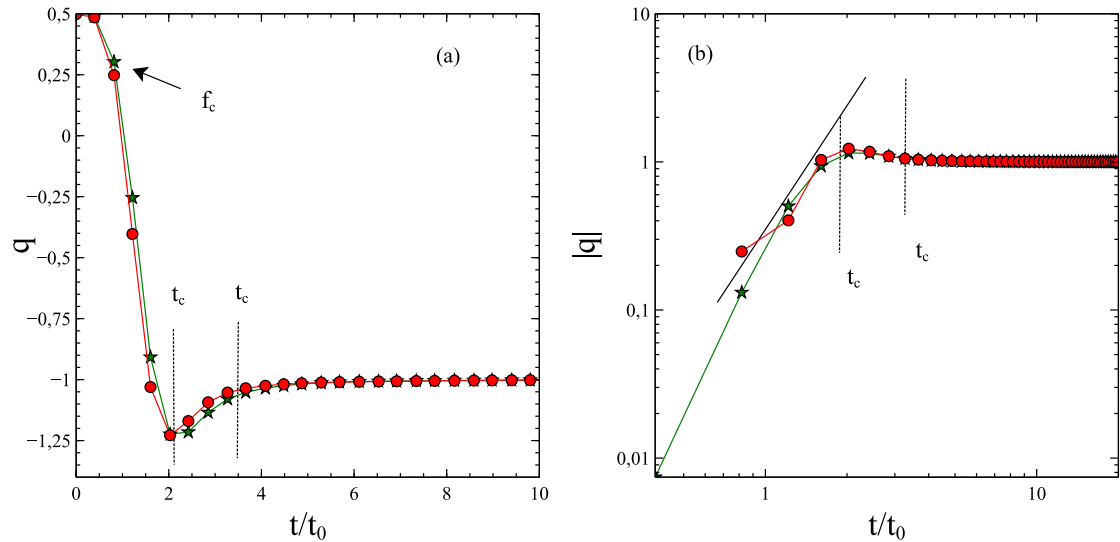

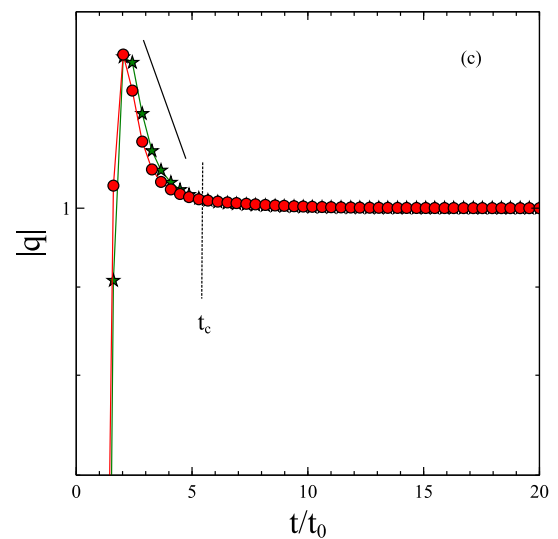

Fig. 4 The deceleration parameter $q$ with respect to cosmic time is displayed. Here we set the parameter values $\Lambda_{1}=0.05, \Lambda_{2}=2.5$, $\Lambda_{3}=0.5, \Lambda_{4}=2.05, A_{1}=A_{4}=1.05, A_{2}=A_{3}=1.5, k_{1}=-1.0$, $k_{2}=1.0$ for the red circle line; $\Lambda_{1}=0.1, \Lambda_{2}=2.6, \Lambda_{3}=0.6$,

eter for the present model is shown in Fig. 4. The time dependent behavior of the deceleration parameter is indicated in Fig. 4a. However, the deceleration parameter is given by log$\log$ and semi-log scale in Fig. $4 \mathrm{~b}$, c, respectively. We note that $\log -\log$ and semi-log figures are plotted for the absolute value of the deceleration parameter after first peaks $f_{c}$ to yield the slope of the curves. Therefore, in Fig. 4b, c, curves occur inversely.

As can be clearly seen that the sign of the phase-like transition also appears at the critical crossover time values $t_{c}$ in Fig. 4a. The deceleration parameter for the early time takes a positive value and rapidly drops to a minimum value at located $t_{c}$ as seen from Fig. 4a. In order to see some details of the time evolution of the deceleration parameter, one can see the Fig. 4b, c. In Fig. 4b, it is seen that the Hubble parameter decreases as $q \propto\left(t / t_{0}\right)^{-\tilde{\beta}}$ up to a critical time point $t_{c}$. On the other hand, after $t_{c}$, it increases exponentially as $h \propto e^{\tilde{\beta}^{\prime}\left(t / t_{0}\right)}$ as seen in Fig. $4 \mathrm{c}$ and it reaches up to a constant value -1 with time for relatively weak and relatively strong interactions where $\tilde{\beta}$, and $\tilde{\beta}^{\prime}$ denote arbitrary constant parameters.

\subsection{The dimensionless jerk parameter $j$}

In cosmology, the dimensionless jerk parameter $j$ corresponds to the acceleration changes of expansion with respect to time. It is a very useful parameter to reveal the hidden transitions between phases of different cosmic accelerations. This parameter is defined as the dimensionless third derivative of the scale factor with respect to cosmic time. To confirm the presence of such a jump in the evolution of the expansion of the Universe, we carry out the presence of the phaselike catastrophic transition for our non-linear interacting two scalar fields model.
$\Lambda_{4}=2.2, A_{1}=A_{4}=1.15, A_{2}=A_{3}=1.6, k_{1}=-1.05, k_{2}=1.0$ for the green star line. In $\mathbf{b}$, deceleration parameter $q$ in Log-Log scale is shown. In c, deceleration parameter $q$ in semi-log plot is provided

The time dependence of the jerk parameter for the present model is displayed in Fig. 5. The time-dependent behavior of the jerk parameter is shown in Fig. 5a. However, the jerk parameter is given by log-log and semi-log scale in Fig. 5b, c, respectively. Notice that the peaks appear at critical crossover times. These cusps strongly indicate a transition in the time evolution of the scale factor $a$. In order to see some detailed time evolution of the jerk parameter around $t_{c}$, we give a $\log -\log$ plot of the jerk parameter, as seen in Fig. 5b. One can observe from this figure that the jerk parameter increases with a power-law exponent $j \propto\left(t / t_{0}\right)^{-\tilde{\gamma}}$ up to critical time point $t_{c}$ and it decays with $j \propto\left(t / t_{0}\right)^{-\tilde{\gamma}^{\prime}}$ where $\gamma$, and $\tilde{\gamma}^{\prime}$ denote arbitrary constant parameters. Finally, after a local minimum value, by decreasing a very weak exponential with time, this parameter reaches up a constant value $j \rightarrow 1$ as well in the $\Lambda$ CDM model as seen Fig. 5c.

\subsection{The dimensionless EoS parameter $w$}

Finally, we study the EoS parameter $w$ as a kinematic variable. The equation of state of a perfect fluid is characterized by a dimensionless number $w$, which is equal to the ratio of its pressure $p$ to its energy density $\rho$. The equation of state may be used in FLRW equations to describe the evolution of an isotropic Universe filled with a perfect fluid. Cosmic inflation and the accelerated expansion of the Universe can be characterized by the equation of state of dark energy and it takes different values for different cosmic eras. In the simplest case, the equation of state of the cosmological constant is $w=-1$. In this case, the scale factor is given by $a(t) \sim \exp \left(H_{0} t\right)$. On the other hand, the EoS parameter can be used to distinguish the phantom and non-phantom dynamics of the Universe. The EoS parameters for the phantom and non-phantom cases are, respectively, given as $w<-1$ and 

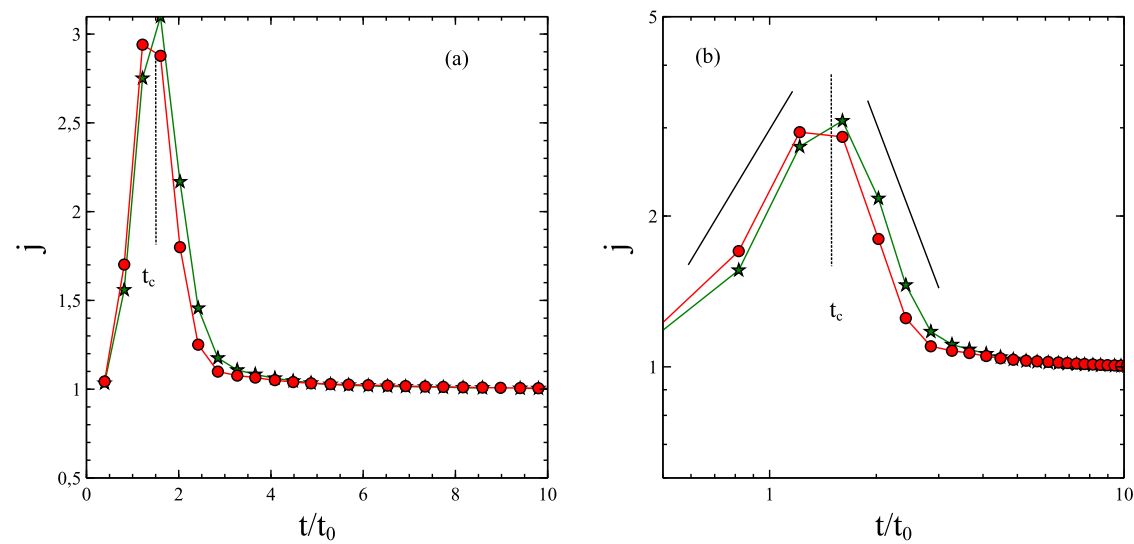

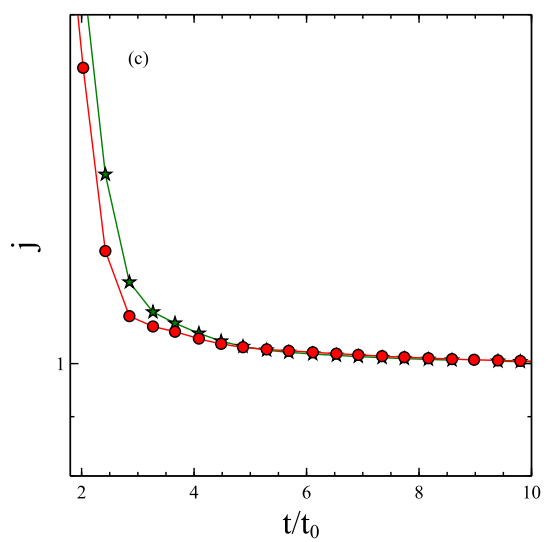

Fig. 5 The jerk parameter $j$ with respect to cosmic time. Here we set the parameter values $\Lambda_{1}=0.05, \Lambda_{2}=2.5, \Lambda_{3}=0.5, \Lambda_{4}=2.05$, $A_{1}=A_{4}=1.05, A_{2}=A_{3}=1.5, k_{1}=-1.0, k_{2}=1.0$ for the red circle line; $\Lambda_{1}=0.1, \Lambda_{2}=2.6, \Lambda_{3}=0.6, \Lambda_{4}=2.2, A_{1}=A_{4}=1.15$,
$A_{2}=A_{3}=1.6, k_{1}=-1.05, k_{2}=1.0$ for the green star line. In $\mathbf{b}$, jerk parameter $j$ in $\log -\log$ scale is provided. In $\mathbf{c}$, jerk parameter $j$ in semi-log plot is shown

the single formula by

$a(t)=a_{0}\left(t / t_{0}\right)^{2 / 3} e^{h\left(t / t_{0}\right)}$,

where $a_{0}$ is the normalization constant. Now we can derive other quantities due to scale factor $a$. The cosmological parameters including Hubble parameter, deceleration, jerk and EoS parameter are respectively given by

$$
\begin{aligned}
h & =\frac{\dot{a}}{a}=\frac{2}{3}\left(t / t_{0}\right)^{-1}+h_{0}, \\
q & =-\frac{\ddot{a}}{a h^{2}}=-1+\frac{6}{\left(2+3 h t / t_{0}\right)^{2}}, \\
j & =\frac{\dddot{a}}{a h^{3}}=1+\frac{36}{\left(2+3 h t / t_{0}\right)^{3}}+\frac{18}{\left(2+3 h t / t_{0}\right)^{2}}, \\
w & =-\frac{1}{3}-\frac{2 a \ddot{a}}{3 \dot{a}^{2}}=-1+\frac{4}{\left(2+3 h t / t_{0}\right)^{2}} .
\end{aligned}
$$

where $h_{0}$ is a constant. It is clear that one obviously obtains power-law and exponential law expansion from Eq. (29) in the limiting cases. Accordingly, for $t / t_{0} \rightarrow 0$, i.e. $t / t_{0} \leq t_{c}$, the cosmological parameters approximate to the following:

$$
\begin{gathered}
a=a_{0}\left(t / t_{0}\right)^{2 / 3}, h \sim \frac{2}{3}\left(t / t_{0}\right)^{-1}, \\
q \sim 1 / 2, j \sim 1 w \sim 0 .
\end{gathered}
$$

Similarly, the exponential term dominates at late times, such that in the limit $t / t_{0} \rightarrow \infty$, i.e. $t / t_{0}>t_{c}$, we have

$a=a_{0} e^{h t / t_{0}}, h \rightarrow h_{0}, q \rightarrow-1, j \rightarrow 1, w \rightarrow-1$

Notice that our results are consistent with the theoretical predictions and observational data in the limiting cases. solving numerically. We see that scale factor can be given in 

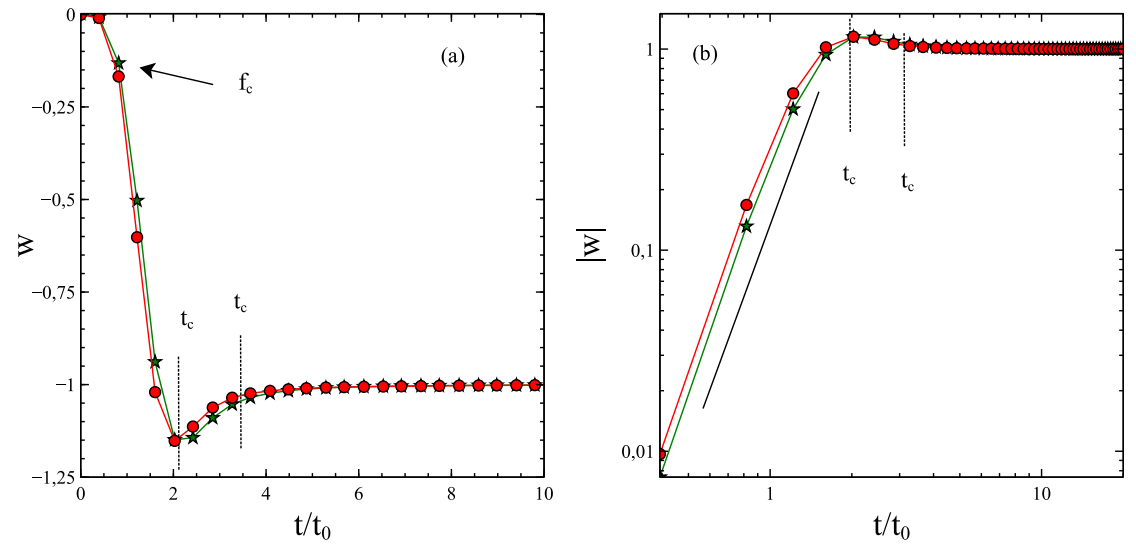

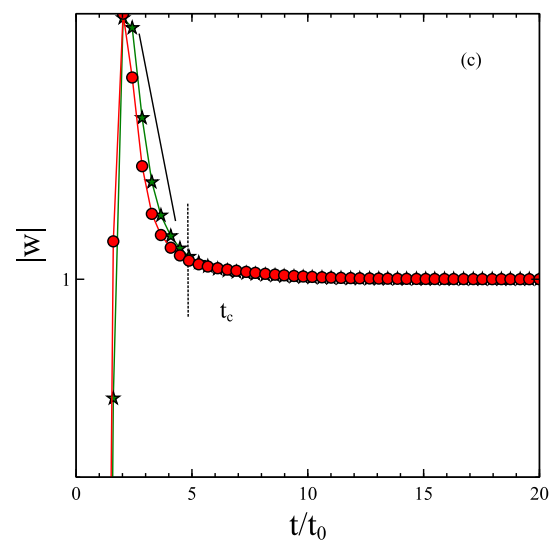

$A_{2}=A_{3}=1.6, k_{1}=-1.05, k_{2}=1.0$ for the green star line is given. In $\mathbf{b}$, EoS parameter $w$ in Log-Log scale. In c, EoS parameter $w$ in semi-log plot is shown

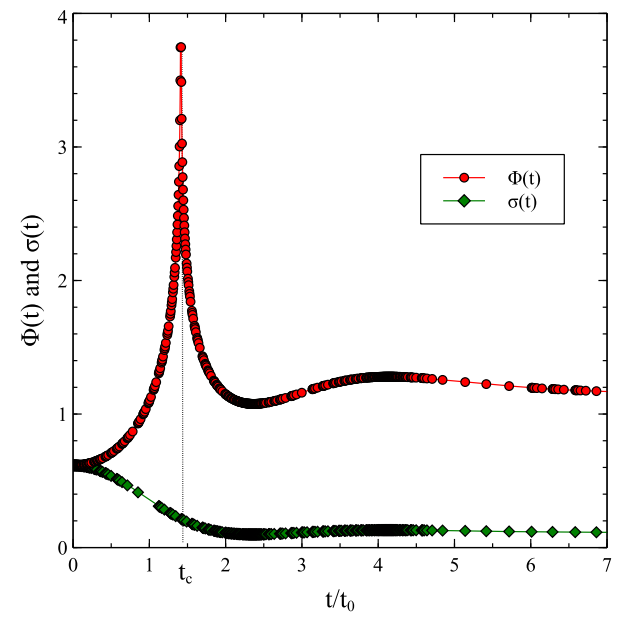

Fig. 7 Time dependent behavior of the scalar fields. Scalar field $\phi(t)$ and $\sigma(t)$ corresponds to the dark energy and dark matter, respectively. Here we set the parameter values $\Lambda_{1}=0.05, \Lambda_{2}=2.5, \Lambda_{3}=0.5$, $\Lambda_{4}=2.05, A_{1}=A_{4}=1.05, A_{2}=A_{3}=1.5, k_{1}=-1.0, k_{2}=1.0$

ferently with time. They reach different saturate values in the late universe period (and in the future). This clearly indicates that dark energy will play a more dominant role in the late time era of the universe. At the same, time, these results support that both scalar fields play an important role in the beginning. We know from the literature that many models based on dark energy have been proposed to explain early inflation of the universe. These models successfully explain the early dynamics [78]. Our findings are also consistent with the theoretical predictions of the other models [78].

In principle, the scalar fields can be written in terms of each other [20,78-80]. This method has been used in some models such as $\Lambda \mathrm{CDM}$, the generalized Chaplygin gas, interacting and phantom models [78]. It would also be interesting 
to use this approach. However, in the present work, we consider both fields together and we restricted ourselves to study the transition from the matter-dominant to the dark energydominated era.

Finally we note that, in order to get a better picture about early and future time dynamics of the universe, the present model should be extended to the multiple (at least three) scalar fields. Additionally, some open problems in the present work such as the energy densities, phase space analysis of the interacting potential, multiple scalar fields model and investigation of the early universe period and the transition from radiation to the matter-dominated era need to be the further study.

\section{Conclusion}

In the present work, we introduce a cosmology model to explain the physical mechanism of the transition from the matter-dominated to dark energy-dominated era and to find a hybrid scale factor that covers both periods. Therefore, we consider an interacting Lagrangian where two scalar fields correspond to the dark matter and dark energy interaction in the framework of the FLRW metric. We assume that two scalar fields interact with a potential which is determined by two oscillators and two anti-oscillators.

We analytically solve the field equations in the FLRW framework for this model and obtain an exact form of the scale factor. We numerically analyze the scale factor and give in Fig. 2. We show that our numerical result produces a hybrid scale factor incorporating the power and exponential terms as $a(t)=a_{0}\left(t / t_{0}\right)^{\alpha} e^{h t / t_{0}}$. This main and significant result clearly denotes that there is a crossover at $t_{c}$. Below $t / t_{0} \leq t_{c}$, the evolution of the Universe is dominated by the matter with a scale factor $a(t) \propto\left(t / t_{0}\right)^{\alpha}$, on the other hand, above $t / t_{0}>t_{c}$, the evolution is dominated by the dark energy with a scale factor $a(t) \propto \exp \left(h t / t_{0}\right)$.

Furthermore, surprisingly, we find that the scale factor behaves as $a(t) \propto\left(t / t_{0}\right)^{2 / 3}$ below $t / t_{0} \leq t_{c}$, and as $a(t) \propto e^{h\left(t / t_{0}\right)}$ within the interval of around $H_{0}=69.5$ and $H_{0}=73.5 \mathrm{~km} \mathrm{~s}^{-1} \mathrm{Mpc}^{-1}$, which shows the dependence on the weak and strong interactions between dark components above $t / t_{0}>t_{c}$, respectively. The exponent $\alpha=2 / 3$ and transition point $t_{0} \simeq 9.8$ Gyear in cosmic time are completely consistent with exact solutions of the FLRW and observations [55]. It is very consistent with the theoretical solutions [54] and time-dependent Hubble parameter $H_{0}$ takes value in the observable intervals given by CMB with Planck [56-58], CMB without Planck [59-61], No CMB, with BBN [62-64], Cepheids-SNIa [65-74], TRGB-SNIa [81-86], Masers [87], Tully-Fisher Relation [88,89], Surface Brightness Fluctuations [90], Lensing related, mass model-dependent [91-98],
Optimistic average [99] and Ultra conservative, no Cepheids, no lensing [100] (for more references please see Ref. [75]).

Additionally, we numerically obtain other dimensionless quantities such as little Hubble $h$, deceleration $q$, jerk parameter $j$ and $\operatorname{EoS} w$ by using scale factor $a(t)$ in Figs. 3, 4, 5 and 6 , respectively. These parameters reflect different aspects of all information on the scale factor since they are obtained depending on the scale factor and/or its derivatives. Indeed, as one can notice from the relevant descriptions of figures leading to the crossover, late time transition from power-law to the exponential one is obtained. All obtained numerical results are consistent with the observational data and theoretical studies.

One can see that the presented model yields very compatible results with the cosmological observations and theoretical expectations. We remark that the choice for the values of free parameters of the potential which involves the catastrophic-like terms do not destroy the characteristic of the solutions since we adjust the parameter values to satisfy the zero energy condition. We see that the results are only dependent on the potential structure and they do not vary considerably when the parameters are changed. This small change could be safely ignored because they arise due to small variations of the zero energy conditions.

In summary, our results clearly show: (i) the late time transition can be modeled using an interacting cosmology model, (ii) a hybrid scale factor can be analytically obtained from this model, (iii) the model precisely predicts the time dependence of the evolution for the matter and dark energy dominated eras, (iv) the model approximately predicts time crossover point in the cosmic time-line between two different cosmological eras, (v) dark energy plays dominant role on the time evolution of the universe. These outstanding findings clearly imply that the interactions in the dark sector can play an important role in understanding of the time evolution of the Universe and other problems of the physical cosmology.

Finally, with this study we conclude that interacting cosmology models have the potential to solve the problems of physical cosmology such as singularity, cosmic coincidence, time evolution of the universe, future and end of the universe, and so on [29-52]. Therefore, we state that the interacting cosmology models deserve more attention for further studies since they can be generalized to many linear or non-linear interacting components. With this motivation, we believe that the method presented in this study provides a good mathematical tool to study Chaotic Universe Theory [52] to obtain metric-dependent solutions and discuss the open problems mentioned above. This is an issue that we will deal with in the future.

Acknowledgements E. A. is grateful to the referee and Editor for the valuable questions and comments which contribute to improve the quality of the manuscript. This work was supported by Istanbul Univer- 
sity Post-Doctoral Research Project: MAB-2019-33386 which is titled "The metric dependent investigation of Chaotic Universe Theory" and Research Project: FYO-2021-38105 which is titled "Investigation of the Cosmic Evolution of the Universe". I. B.-Ö. grateful to Istanbul University for hospitality and grant for the Post-Doctoral Researcher Position under supervisor E. A. at Istanbul University.

Author contributions E. A. and T. D. developed the theory. All coauthors performed to obtain the analytical solutions. E. A. and I. B.O. obtained the numerical results and plotted figures. E. A and T. D. interpreted the initial results. E. A. interpreted all results and wrote the manuscript.

Data Availability Statement This manuscript has no associated data or the data will not be deposited. [Authors' comment: This is a theoretical study and no experimental data has been listed.]

Open Access This article is licensed under a Creative Commons Attribution 4.0 International License, which permits use, sharing, adaptation, distribution and reproduction in any medium or format, as long as you give appropriate credit to the original author(s) and the source, provide a link to the Creative Commons licence, and indicate if changes were made. The images or other third party material in this article are included in the article's Creative Commons licence, unless indicated otherwise in a credit line to the material. If material is not included in the article's Creative Commons licence and your intended use is not permitted by statutory regulation or exceeds the permitted use, you will need to obtain permission directly from the copyright holder. To view a copy of this licence, visit http://creativecomm ons.org/licenses/by/4.0/.

Funded by SCOAP ${ }^{3}$.

\section{References}

1. A.G. Riess, A.V. Filippenko, P. Challis, A. Clocchiatti, A. Diercks, P.M. Garnavich, R.L. Gilliland, C.J. Hogan, S. Jha, R.P. Kirshner, B. Leibundgut, M.M. Phillips, D. Reiss, B.P. Schmidt, R.A. Schommer, R.C. Smith, J. Spyromilio, C. Stubbs, N.B. Suntzeff, J. Tonry, Observational evidence from supernovae for an accelerating universe and a cosmological constant. Astron. J. 116, 1009 (1998). https://doi.org/10.1086/300499 arXiv:astro-ph/9805201

2. S. Perlmutter, G. Aldering, G. Goldhaber, R.A. Knop, P. Nugent, P.G. Castro, S. Deustua, S. Fabbro, A. Goobar, D. E. Groom, I.M. Hook, A.G. Kim, M.Y. Kim, J.C. Lee, N.J. Nunes, R. Pain, C.R. Pennypacker, R. Quimby, C. Lidman, R.S. Ellis, M. Irwin, R.G. McMahon, P. Ruiz-Lapuente, N. Walton, B. Schaefer, B.J. Boyle, A.V. Filippenko, T. Matheson, A.S. Fruchter, N. Panagia, H.J.M. Newberg, W.J. Couch, T.S.C. Project, Measurements of $\omega$ and $\lambda$ from 42 high redshift supernovae. Astrophys. J. 517, 565 (1999). https://doi.org/10.1086/307221

3. T. Barreiro, E.J. Copeland, N.J. Nunes, Quintessence arising from exponential potentials. Phys. Rev. D 61, 127301 (2000). https:// doi.org/10.1103/PhysRevD.61.127301 arXiv:astro-ph/9910214

4. R.R. Caldwell, A phantom menace? Cosmological consequences of a dark energy component with super-negative equation of state. Phys. Lett. B 545, 23 (2002). https://doi.org/10.1016/ S0370-2693(02)02589-3 arXiv:astro-ph/9908168

5. C. Armendariz-Picon, V. Mukhanov, P.J. Steinhardt, Essentials of k-essence. Phys. Rev. D 63, 103510 (2001). https://doi.org/10. 1103/PhysRevD.63.103510 arXiv:astro-ph/0006373

6. J.S. Bagla, H.K. Jassal, T. Padmanabhan, Cosmology with tachyon field as dark energy. Phys. Rev. D 67,
063504 (2003). https://doi.org/10.1103/PhysRevD.67.063504 arXiv:astro-ph/0212198

7. M.C. Bento, O. Bertolami, A.A. Sen, Generalized Chaplygin gas, accelerated expansion, and dark-energy-matter unification. Phys. Rev. D 66, 043507 (2002). https://doi.org/10.1103/PhysRevD.66. 043507

8. M. Li, A model of holographic dark energy. Phys. Lett. B 603, 1 (2004). https://doi.org/10.1016/j.physletb.2004.10.014 arXiv:hep-th/0403127

9. R. Thom, Structural Stability and Morphogenesis: An Outline of a General Theory of Models, Advanced book classics (CRC Press, Taylor \& Francis GroupPerseus Books, 1989). http://gen.lib.rus. ec/book/index.php?md5=dc2fb0e837fc2fe0f3b3dfc2c972187f

10. A.D. Linde, A new inflationary universe scenario: a possible solution of the horizon, flatness, homogeneity, isotropy and primordial monopole problems. Phys. Lett. B 108, 389 (1982). https://doi. org/10.1016/0370-2693(82)91219-9

11. S. Weinberg, Cosmology (Oxford University Press, USA, 2008). http://gen.lib.rus.ec/book/index.php? md5=5a9fb4ef1fe319a5b02fdac17ddfea94

12. S. Capozziello, M. Roshan, Exact cosmological solutions from Hojman conservation quantities. Phys. Lett. B 726, 471 (2013). https://doi.org/10.1016/j.physletb.2013.08.047 arXiv:1308.3910 [gr-qc]

13. J.A. Belinchón, T. Harko, M.K. Mak, Exact scalar-tensor cosmological models. Int. J. Mod. Phys. D 26, 1750073 (2017). https:// doi.org/10.1142/S0218271817500730 arXiv:1612.05446 [gr-qc]

14. B. Tajahmad, Studying the intervention of an unusual term in $f(t)$ gravity via the Noether symmetry approach. Eur. Phys. J. C 77, 510 (2017). https://doi.org/10.1140/epjc/s10052-017-5050-z arXiv:1701.01620 [gr-qc]

15. M. Sharif, I. Nawazish, Cosmological analysis of scalar field models in $f(r, t)$ gravity. Eur. Phys. J. C 77, 198 (2017). https://doi. org/10.1140/epjc/s10052-017-4773-1 arXiv:1703.06763 [gr-qc]

16. A. Paliathanasis, G. Leon, Analytic solutions in Einstein-Aether scalar field cosmology. Eur. Phys. J. C 80, 355 (2020). https://doi. org/10.1140/epjc/s10052-020-7924-8 arXiv:2003.03903 [gr-qc]

17. A. Paliathanasis, M. Tsamparlis, Two scalar field cosmology: conservation laws and exact solutions. Phys. Rev. D 90, 043529 (2014). https://doi.org/10.1103/PhysRevD.90.043529 arXiv: 1408.1798 [gr-qc]

18. Y. Kucukakca, A.R. Akbarieh, Noether symmetries of EinsteinAether scalar field cosmology. Eur. Phys. J. C 80, 1019 (2020). https://doi.org/10.1140/epjc/s10052-020-08583-7

19. S. Nojiri, S. Odintsov, V. Oikonomou, Modified gravity theories on a nutshell: inflation, bounce and late-time evolution. Phys. Rep. 692, 1 (2017). https://doi.org/10.1016/j.physrep.2017.06.001

20. S. Nojiri, S. Odintsov, Unifying phantom inflation with late-time acceleration: scalar phantom-non-phantom transition model and generalized holographic dark energy. Gen. Relativ. Gravit. 38, 1572 (2006) https://doi.org/10.1007/s10714-006-0301-6

21. I. Oz, Y. Kucukakca, N. Unal, Anisotropic solution in phantom cosmology via Noether symmetry approach1. Can. J. Phys. 96, 677 (2018). https://doi.org/10.1139/cjp-2017-0765

22. S. Bahamonde, C.G. Böhmer, S. Carloni, E.J. Copeland, W. Fang, N. Tamanini, Dynamical systems applied to cosmology: dark energy and modified gravity. Phys. Rep. 775-777, 1 (2018) https:// doi.org/10.1016/j.physrep.2018.09.001

23. R.R. Caldwell, R. Dave, P.J. Steinhardt, Cosmological imprint of an energy component with general equation of state. Phys. Rev. Lett. 80, 1582 (1998). https://doi.org/10.1103/PhysRevLett.80. 1582 arXiv:astro-ph/9708069

24. A.R. Liddle, R.J. Scherrer, Classification of scalar field potentials with cosmological scaling solutions. Phys. Rev. D 59, 023509 (1998). https://doi.org/10.1103/PhysRevD.59.023509 arXiv:astro-ph/9809272 
25. P. Peebles, B. Ratra, The cosmological constant and dark energy. Rev. Mod. Phys. 75, 559 (2003). https://doi.org/10.1103/ RevModPhys.75.559 arXiv:astro-ph/0207347

26. C. Wetterich, Cosmology and the fate of dilatation symmetry. Nucl. Phys. B 302, 668 (1988). https://doi.org/10.1016/ 0550-3213(88)90193-9 arXiv:1711.03844 [hep-th]

27. J.D. Barrow, Quiescent cosmology. Nature 272, 211 (1978). https://doi.org/10.1038/272211a0

28. P. Brax, J. Martin, The supergravity quintessence model coupled to the minimal supersymmetric standard model. J. Cosmol. Astropart. Phys. 11, 008. https://doi.org/10.1088/1475-7516/ 2006/11/008. arXiv:astro-ph/0606306

29. Y.L. Bolotin, A. Kostenko, O. Lemets, D. Yerokhin, Cosmological evolution with interaction between dark energy and dark matter. Int. J. Mod. Phys. D 24, 1530007 (2015). https://doi.org/10.1142/ S0218271815300074 arXiv:1310.0085 [astro-ph.CO]

30. W. Zimdahl, D. Pavon, Statefinder parameters for interacting dark energy. Gen. Relativ. Gravit. 36, 1483-1491 (2004). https://doi.org/10.1023/B:GERG.0000022584.54115.9e arXiv:gr-qc/0311067

31. L. Amendola, G.C. Campos, R. Rosenfeld, Consequences of dark matter-dark energy interaction on cosmological parameters derived from type Ia supernova data. Phys. Rev. D 75, 083506 (2007). https://doi.org/10.1103/PhysRevD.75.083506 arXiv:astro-ph/0610806

32. B. Wang, E. Abdalla, F. Atrio-Barandela, D. Pavon, Dark matter and dark energy interactions: theoretical challenges, cosmological implications and observational signatures. Rep. Prog. Phys. 79, 096901 (2016). https://doi.org/10.1088/0034-4885/79/9/096901 arXiv:1603.08299 [astro-ph.CO]

33. C.G. Böhmer, N. Tamanini, M. Wright, Interacting quintessence from a variational approach. I. algebraic couplings. Phys. Rev. D 91, 123002 (2015). https://doi.org/10.1103/PhysRevD.91. 123002. arXiv: 1501.06540 [gr-qc]

34. C.G. Böhmer, N. Tamanini, M. Wright, Interacting quintessence from a variational approach. II. Derivative couplings. Phys. Rev. D 91, 123003 (2015). https://doi.org/10.1103/PhysRevD.91. 123003. arXiv: 1502.04030 [gr-qc]

35. J.-H. He, B. Wang, Effects of the interaction between dark energy and dark matter on cosmological parameters. J. Cosmol. Astropart. Phys. 06, 010. https://doi.org/10.1088/1475-7516/ 2008/06/010. arXiv:70801.4233 [astro-ph]

36. R.-G. Cai, N. Tamanini, T. Yang, Reconstructing the dark sector interaction with LISA. J. Cosmol. Astropart. Phys. 05, 031. https://doi.org/10.1088/1475-7516/2017/05/031. arXiv:1703.07323 [astro-ph.CO]

37. W. Yang, N. Banerjee, A. Paliathanasis, S. Pan, Reconstructing the dark matter and dark energy interaction scenarios from observations. Phys. Dark Universe 26, 100383 (2019). https://doi.org/ 10.1016/j.dark.2019.100383 arXiv:1812.06854 [astro-ph.CO]

38. L. Amendola, Coupled quintessence. Phys. Rev. D 62, 043511 (2000). https://doi.org/10.1103/PhysRevD.62.043511 arXiv:astro-ph/9908023

39. D. Tocchini-Valentini, L. Amendola, Stationary dark energy with a baryon-dominated era: solving the coincidence problem with a linear coupling. Phys. Rev. D 65, 063508 (2002). https://doi.org/ 10.1103/PhysRevD.65.063508 arXiv:astro-ph/0108143

40. L. Amendola, C. Quercellini, Tracking and coupled dark energy as seen by the Wilkinson microwave anisotropy probe. Phys. Rev. D 68, 023514 (2003). https://doi.org/10.1103/PhysRevD.68. 023514 arXiv:astro-ph/0303228

41. S. del Campo, R. Herrera, D. Pavon, Toward a solution of the coincidence problem. Phys. Rev. D 78, 021302 (2008). https://doi. org/10.1103/PhysRevD.78.021302 arXiv:0806.2116 [astro-ph]

42. S. del Campo, R. Herrera, D. Pavon, Interacting models may be key to solve the cosmic coincidence problem. J. Cosmol.
Astropart. Phys. (01), 020. https://doi.org/10.1088/1475-7516/ 2009/01/020. arXiv:0812.2210 [gr-qc]

43. H. Wei, S.N. Zhang, Observational $\mathrm{h}(\mathrm{z})$ data and cosmological models. Phys. Lett. B 644, 7 (2007). https://doi.org/10.1016/j. physletb.2006.11.027 arXiv:astro-ph/0609597

44. S. del Campo, R. Herrera, D. Pavón, Interaction in the dark sector. Phys. Rev. D 91, 123539 (2015). https://doi.org/10.1103/ PhysRevD.91.123539 arXiv:1507.00187 [gr-qc]

45. L.P. Chimento, Linear and nonlinear interactions in the dark sector. Phys. Rev. D 81, 043525 (2010). https://doi.org/10.1103/ PhysRevD.81.043525 arXiv:0911.5687 [astro-ph.CO]

46. G. Sanchez, E. Ivan, Dark matter interacts with variable vacuum energy. Gen. Relativ. Gravit. 46, 1769 (2014). https://doi.org/10. 1007/s10714-014-1769-0 arXiv:1405.1291 [gr-qc]

47. M.M. Verma, Dark energy as a manifestation of the non-constant cosmological constant. Astrophys. Space Sci. 330, 101 (2010). https://doi.org/10.1007/s10509-010-0347-5

48. M. Shahalam, S.D. Pathak, M.M. Verma, M.Y. Khlopov, R. Myrzakulov, Dynamics of interacting quintessence. Eur. Phys. J. C 75, 395 (2015). https://doi.org/10.1140/epjc/ s10052-015-3608-1 arXiv:1503.08712 [gr-qc]

49. M. Cruz, S. Lepe, Holographic approach for dark energy-dark matter interaction in curved FLRW spacetime. Class. Quantum Gravity 35, 155013 (2018). https://doi.org/10.1088/1361-6382/ aacd9e

50. M. Cruz, S. Lepe, G. Morales-Navarrete, Qualitative description of the universe in the interacting fluids scheme. Nucl. Phys. B 943, 114623 (2019). https://doi.org/10.1016/j.nuclphysb.2019. 114623

51. R. Saleem, M.J. Imtiaz, Dynamical study of interacting Ricci dark energy model using Chevallier-Polarsky-Lindertype parametrization. Class. Quantum Gravity 37, 065018 (2020). https://doi.org/10.1088/1361-6382/ab6f0f

52. E. Aydiner, Chaotic universe model. Sci. Rep. 8, 721 (2018). https://doi.org/10.1038/s41598-017-18681-4

53. T. Dereli, R.W. Tucker, Signature dynamics in general relativity. Class. Quantum Gravity 10, 365 (1993). https://doi.org/10.1088/ 0264-9381/10/2/018

54. A. Einstein, W. de Sitter, On the relation between the expansion and the mean density of the universe. Proc. Natl. Acad. Sci. 18, 213 (1932). https://doi.org/10.1073/pnas.18.3.213. https://www. pnas.org/content/18/3/213.full.pdf

55. B. Ryden, Introduction to Cosmology, 2nd edn. (Cambridge University Press, New York, 2016)

56. L. Balkenhol et al. (SPT), Constraints on $\Lambda \mathrm{CDM}$ extensions from the SPT-3G $2018 E E$ and $T E$ Power Spectra (2021). arXiv:2103.13618 [astro-ph.CO]

57. N. Aghanim et al. (Planck), Planck 2018 results. VI. Cosmological parameters, Astron. Astrophys. 641, A6 (2020). https://doi. org/10.1051/0004-6361/201833910. arXiv:1807.06209 [astroph.CO]

58. N. Aghanim et al. (Planck), Planck 2018 results. V. $\mathrm{CMB}$ power spectra and likelihoods. Astron. Astrophys. 641, A5 (2020). https://doi.org/10.1051/0004-6361/201936386. arXiv:1907.12875 [astro-ph.CO]

59. D. Dutcher et al. (SPT-3G), Measurements of the E-Mode Polarization and Temperature-E-Mode Correlation of the CMB from SPT-3G 2018 Data (2021). arXiv:2101.01684 [astro-ph.CO]

60. S. Aiola et al. (ACT), The Atacama Cosmology Telescope: DR4 maps and cosmological parameters. JCAP 12, 047. https://doi. org/10.1088/1475-7516/2020/12/047. arXiv:2007.07288 [astroph.CO]

61. X. Zhang, Q.-G. Huang, Constraints on $H_{0}$ from WMAP and BAO measurements. Commun. Theor. Phys. 71, 826 (2019). https:// doi.org/10.1088/0253-6102/71/7/826 arXiv:1812.01877 [astroph.CO] 
62. O.H.E. Philcox, M.M. Ivanov, M. Simonović, M. Zaldarriaga, Combining full-shape and $\mathrm{BAO}$ analyses of galaxy power spectra: a $1.6 \% \mathrm{CMB}$-independent constraint on $\mathrm{H}_{0}$. JCAP 05, 032. https://doi.org/10.1088/1475-7516/2020/05/032. arXiv:2002.04035 [astro-ph.CO]

63. M.M. Ivanov, M. Simonović, M. Zaldarriaga, Cosmological parameters from the BOSS Galaxy Power Spectrum. JCAP 05, 042. https://doi.org/10.1088/1475-7516/2020/05/042. arXiv:1909.05277 [astro-ph.CO]

64. S. Alam et al., (eBOSS), Completed SDSS-IV extended baryon oscillation spectroscopic survey: cosmological implications from two decades of spectroscopic surveys at the Apache Point Observatory. Phys. Rev. D 103, 083533 (2021). https://doi.org/10.1103/ PhysRevD.103.083533 arXiv:2007.08991 [astro-ph.CO]

65. A.G. Riess, S. Casertano, W. Yuan, J.B. Bowers, L. Macri, J.C. Zinn, D. Scolnic, Cosmic distances calibrated to $1 \%$ precision with Gaia EDR3 parallaxes and Hubble Space Telescope Photometry of 75 Milky Way Cepheids Confirm Tension with $\Lambda$ CDM. Astrophys. J. Lett. 908, L6 (2021). https://doi.org/10. 3847/2041-8213/abdbaf arXiv:2012.08534 [astro-ph.CO]

66. L. Breuval et al., The Milky Way Cepheid Leavitt law based on Gaia DR2 parallaxes of companion stars and host open cluster populations. Astron. Astrophys. 643, A115 (2020). https://doi. org/10.1051/0004-6361/202038633 arXiv:2006.08763 [astroph.SR]

67. A.G. Riess, S. Casertano, W. Yuan, L.M. Macri, D. Scolnic, Large magellanic cloud Cepheid standards provide a $1 \%$ foundation for the determination of the Hubble constant and stronger evidence for physics beyond $\Lambda$ CDM. Astrophys. J. 876, 85 (2019). https://doi.org/10.3847/1538-4357/ab1422 arXiv:1903.07603 [astro-ph.CO]

68. D. Camarena, V. Marra, Local determination of the Hubble constant and the deceleration parameter. Phys. Rev. Res. 2, 013028 (2020). https://doi.org/10.1103/PhysRevResearch.2. 013028 arXiv:1906.11814 [astro-ph.CO]

69. C.R. Burns et al., (CSP), The Carnegie Supernova Project: absolute calibration and the Hubble constant. Astrophys. J. 869, 56 (2018). https://doi.org/10.3847/1538-4357/aae51c arXiv:1809.06381 [astro-ph.CO]

70. B. Follin, L. Knox, Insensitivity of the distance ladder Hubble constant determination to Cepheid calibration modelling choices. Mon. Not. R. Astron. Soc. 477, 4534 (2018). https://doi.org/10. 1093/mnras/sty720 arXiv:1707.01175 [astro-ph.CO]

71. S.M. Feeney, D.J. Mortlock, N. Dalmasso, Clarifying the Hubble constant tension with a Bayesian hierarchical model of the local distance ladder. Mon. Not. R. Astron. Soc. 476, 3861 (2018). https://doi.org/10.1093/mnras/sty418 arXiv:1707.00007 [astroph.CO]

72. A.G. Riess et al., A $2.4 \%$ determination of the local value of the Hubble constant. Astrophys. J. 826, 56 (2016). https://doi.org/10. 3847/0004-637X/826/1/56. arXiv:1604.01424 [astro-ph.CO]

73. W. Cardona, M. Kunz, V. Pettorino, Determining $H_{0}$ with Bayesian hyper-parameters, JCAP 03, 056. https://doi.org/10. 1088/1475-7516/2017/03/056. arXiv:1611.06088 [astro-ph.CO]

74. W.L. Freedman, B.F. Madore, V. Scowcroft, C. Burns, A. Monson, S.E. Persson, M. Seibert, J. Rigby, Carnegie Hubble Program: a mid-infrared calibration of the Hubble constant. Astrophys. J. 758, 24 (2012). https://doi.org/10.1088/0004-637X/758/ 1/24 arXiv:1208.3281 [astro-ph.CO]

75. E. Di Valentino, O. Mena, S. Pan, L. Visinelli, W. Yang, A. Melchiorri, D.F. Mota, A.G. Riess, J. Silk, In the realm of the Hubble tension-a review of solutions (2021). https://doi.org/10.1088/ 1361-6382/ac086d. arXiv:2103.01183 [astro-ph.CO]

76. A.G. Riess, L. Macri, S. Casertano, M. Sosey, H. Lampeitl, H.C. Ferguson, A.V. Filippenko, S.W. Jha, W. Li, R. Chornock, D. Sarkar, A redetermination of the Hubble constant with the Hubble space telescope from a differerntial distance ladder. Astrophys. J. 699, 539 (2009). https://doi.org/10.1088/0004-637x/699/1/539

77. D.J. Croton, Damn you, little h! (or, real-world applications of the Hubble constant using observed and simulated data). Publ. Astron. Soc. Aust. 30, e052 (2013). https://doi.org/10.1017/pasa. 2013.31

78. K. Bamba, S. Capozziello, S. Nojiri, S.D. Odintsov, Dark energy cosmology: the equivalent description via different theoretical models and cosmography tests. Astrophys. Space Sci. 342, 155 (2012). https://doi.org/10.1007/s10509-012-1181-8

79. S. Capozziello, S. Nojiri, S. Odintsov, Dark energy: the equation of state description versus scalar-tensor or modified gravity. Phys. Lett. B 634, 93 (2006). https://doi.org/10.1016/j.physletb.2006. 01.065

80. S. Capozziello, S. Nojiri, S. Odintsov, Unified phantom cosmology: inflation, dark energy and dark matter under the same standard. Phys. Lett. B 632, 597 (2006). https://doi.org/10.1016/j. physletb.2005.11.012

81. J. Soltis, S. Casertano, A.G. Riess, The Parallax of $\omega$ Centauri measured from Gaia EDR3 and a direct, geometric calibration of the tip of the red giant branch and the Hubble constant. Astrophys. J. Lett. 908, L5 (2021). https://doi.org/10.3847/2041-8213/ abdbad arXiv:2012.09196 [astro-ph.GA]

82. W.L. Freedman, B.F. Madore, T. Hoyt, I.S. Jang, R. Beaton, M.G. Lee, A. Monson, J. Neeley, J. Rich, Calibration of the Tip of the Red Giant Branch (TRGB) (2020). https://doi.org/10.3847/ 1538-4357/ab7339. arXiv:2002.01550 [astro-ph.GA]

83. M.J. Reid, D.W. Pesce, A.G. Riess, An improved distance to NGC 4258 and its implications for the Hubble constant. Astrophys. J. Lett. 886, L27 (2019). https://doi.org/10.3847/2041-8213/ ab552d arXiv:1908.05625 [astro-ph.GA]

84. W.L. Freedman et al., The Carnegie-Chicago Hubble Program. VIII. An independent determination of the hubble constant based on the tip of the Red Giant Branch (2019). https://doi.org/10.3847/ 1538-4357/ab2f73. arXiv:1907.05922 [astro-ph.CO]

85. W. Yuan, A.G. Riess, L.M. Macri, S. Casertano, D. Scolnic, Consistent Calibration of the Tip of the Red Giant Branch in the Large Magellanic Cloud on the Hubble Space Telescope Photometric System and a Re-determination of the Hubble Constant. Astrophys. J. 886, 61 (2019). https://doi.org/10.3847/1538-4357/ ab4bc9 arXiv:1908.00993 [astro-ph.GA]

86. I.S. Jang, M.G. Lee, The Tip of the Red Giant Branch Distances to Typa Ia Supernova Host Galaxies. V. NGC 3021, NGC 3370, and NGC 1309 and the Value of the Hubble Constant. Astrophys. J. 836, 74 (2017). https://doi.org/10.3847/1538-4357/836/1/74. arXiv: 1702.01118 [astro-ph.CO]

87. D.W. Pesce et al., The Megamaser Cosmology Project. XIII. Combined Hubble constant constraints. Astrophys. J. Lett. 891, L1 (2020). https://doi.org/10.3847/2041-8213/ab75f0. arXiv:2001.09213 [astro-ph.CO]

88. E. Kourkchi, R.B. Tully, G.S. Anand, H.M. Courtois, A. Dupuy, J.D. Neill, L. Rizzi, M. Seibert, Cosmicflows-4: the calibration of optical and infrared Tully-Fisher relations. Astrophys. J. 896, 3 (2020). https://doi.org/10.3847/1538-4357/ab901c arXiv:2004.14499 [astro-ph.GA]

89. J. Schombert, S. McGaugh, F. Lelli, Using the baryonic TullyFisher relation to measure H o. Astron. J. 160, 71 (2020). https:// doi.org/10.3847/1538-3881/ab9d88 arXiv:2006.08615 [astroph.CO]

90. J.P. Blakeslee, J.B. Jensen, C.-P. Ma, P.A. Milne, J.E. Greene, The Hubble constant from infrared surface brightness fluctuation distances. Astrophys. J. 911, 65 (2021). https://doi.org/10.3847/ 1538-4357/abe86a arXiv:2101.02221 [astro-ph.CO]

91. M. Millon et al., TDCOSMO. I. An exploration of systematic uncertainties in the inference of $H_{0}$ from time-delay cosmog- 
raphy. Astron. Astrophys. 639, A101 (2020). https://doi.org/10. 1051/0004-6361/201937351. arXiv:1912.08027 [astro-ph.CO]

92. J.-Z. Qi, J.-W. Zhao, S. Cao, M. Biesiada, Y. Liu, Measurements of the Hubble constant and cosmic curvature with quasars: ultracompact radio structure and strong gravitational lensing. Mon. Not. R. Astron. Soc. 503, 2179 (2021). https://doi.org/10.1093/ mnras/stab638 arXiv:2011.00713 [astro-ph.CO]

93. K. Liao, A. Shafieloo, R.E. Keeley, E.V. Linder, Determining model-independent $\mathrm{H} 0$ and consistency tests. Astrophys. J. Lett. 895, L29 (2020). https://doi.org/10.3847/2041-8213/ ab8dbb arXiv:2002.10605 [astro-ph.CO]

94. K. Liao, A. Shafieloo, R.E. Keeley, E.V. Linder, A modelindependent determination of the Hubble constant from lensed quasars and supernovae using Gaussian process regression. Astrophys. J. Lett. 886, L23 (2019). https://doi.org/10.3847/ 2041-8213/ab5308 arXiv:1908.04967 [astro-ph.CO]

95. A.J. Shajib et al. (DES), STRIDES: a 3.9 per cent measurement of the Hubble constant from the strong lens system DES J04085354. Mon. Not. R. Astron. Soc. 494, 6072 (2020). https://doi. org/10.1093/mnras/staa828. arXiv:1910.06306 [astro-ph.CO]

96. K.C. Wong et al., HOLiCOW-XIII. A 2.4 per cent measurement of $\mathrm{H} 0$ from lensed quasars: $5.3 \sigma$ tension between early- and late-Universe probes. Mon. Not. R. Astron. Soc. 498, 1420 (2020). https://doi.org/10.1093/mnras/stz3094. arXiv:1907.04869 [astro-ph.CO]
97. S. Birrer et al., HOLiCOW-IX. Cosmographic analysis of the doubly imaged quasar SDSS $1206+4332$ and a new measurement of the Hubble constant. Mon. Not. R. Astron. Soc. 484, 4726 (2019). https://doi.org/10.1093/mnras/stz200. arXiv:1809.01274 [astro-ph.CO]

98. V. Bonvin et al., HOLiCOW-V. New COSMOGRAIL time delays of HE 0435-1223: $H_{0}$ to 3.8 per cent precision from strong lensing in a flat $\Lambda \mathrm{CDM}$ model. Mon. Not. R. Astron. Soc. 465, 4914 (2017). https://doi.org/10.1093/mnras/stw3006. arXiv:1607.01790 [astro-ph.CO]

99. E. Di Valentino, A. Mukherjee, A.A. Sen, Dark energy with phantom crossing and the $H_{0}$ tension. Entropy 23, 404 (2021). https:// doi.org/10.3390/e23040404 arXiv:2005.12587 [astro-ph.CO]

100. E. Di Valentino, S. Pan, W. Yang, L.A. Anchordoqui, Touch of neutrinos on the vacuum metamorphosis: is the $H_{0}$ solution back? Phys. Rev. D 103, 123527 (2021). https://doi.org/10.1103/ PhysRevD.103.123527 arXiv:2102.05641 [astro-ph.CO] 